\title{
NOTE ON THE FUNDAMENTAL THEOREM ON IRREDUCIBLE NON-NEGATIVE MATRICES
}

\author{
by HANS SCHNEIDER \\ (Received 12th February 1958)
}

1. Let $A=\left[a_{i j}\right]$ be an $n$-th order irreducible non-negative matrix. As is very well-known, the matrix $A$ has a positive characteristic root $\rho$ (provided that $n>1$ ), which is simple and maximal in the sense that every characteristic root $\lambda$ satisfies $|\lambda| \leqslant \rho$, and the characteristic vector $x$ belonging to $\rho$ may be chosen positive. These results, originally due to Frobenius, have been proved by Wielandt (4) by means of a strikingly simple basic idea. Recently, a variant of Wielandt's proof has been given by Householder (2).

We shall sketch part of the proof. For each non-negative column vector $y$ we set

$$
\begin{aligned}
& \rho_{*}(y)=\sup r: r y \leqslant A y, \\
& \rho^{*}(y)=\inf r: r y \geqslant A y .
\end{aligned}
$$

For strictly positive $y$, we may replace (1) and (2) by

$$
\begin{aligned}
& \rho_{*}(y)=\min _{i} \frac{(A y)_{i}}{y_{i}}=\min _{i} \frac{\sum_{j=1}^{n} a_{i j} y_{j}}{y_{i}}, \\
& \rho^{*}(y)=\max _{i} \frac{(A y)_{i}}{y_{i}}=\max _{i} \frac{\sum_{j=1}^{n} a_{i j} y_{j} .}{y_{i}}
\end{aligned}
$$

Let $P$ be the section of the non-negative cone (all $y_{i} \geqslant 0$ ) by the plane $\Sigma_{i=1}^{n} y_{i}=1$. Since $\rho_{*}(\lambda y)=\rho_{*}(y)$, for all positive $\lambda$, the supremum of $\rho_{*}(y)$ over $P$ equals the supremum of $\rho_{*}(y)$ over all $y \geqslant 0$. On $P, \rho_{*}(y)$ attains this supremum, say $\rho=\sup \rho_{*}(y)=\rho_{*}(x)$, where $x$ is on $P$. It is then shown that $A x=\rho x$, that $\rho$ is simple and maximal, and that $x>0$. Similarly the infimum of $\rho^{*}(y)$ over all $y \geqslant 0$ is attained on $P$.

2. In this argument there arises a dilemma :

(i) Either the whole of $P$ is considered, in which case $\rho_{*}(y)$ (or $\rho^{*}(y)$ ) may have singularities and discontinuities at vectors $y$ which have zero elements ;

(ii) Or, the subset $P_{1}$ of $P$, consisting of all positive $y$ on $P$, is considered, in which case $\rho_{*}(y)$ (and $\rho^{*}(y)$ ) are everywhere continuous on $P_{1}$, but $P_{1}$ is not closed.

In either case, some justification is required for the assertion that $\rho_{*}(y)$ attains its supremum (or $\rho^{*}(y)$ its infimum).

For an example of a discontinuity in $\rho_{*}(y)$, examine the case of

$$
A=\left[\begin{array}{lll}
0 & 1 & 0 \\
0 & 0 & 2 \\
1 & 0 & 2
\end{array}\right]
$$


At $z=(0,0,1)$, we have $\rho_{*}(z)=2$. But for all $y$ of form $(\alpha, \alpha, 1-2 \alpha)$ with $0<\alpha \leqslant \frac{1}{4}, \rho_{*}(y)=1$.

It may however be shown that $\rho_{*}(y)$ is upper semi-continuous on $P$, and hence $\rho_{*}(y)$ attains its supremum on $P$. For $\rho^{*}(y)$ slightly more complex reasoning is required. If $R_{1}$ denotes the real line with infinity adjoined, topologised in the normal way, then $\rho^{*}(y)$ is a lower semi-continuous function into $R_{1}$, for all non-negative $A$, and is even continuous when $\mathrm{A}$ is irreducible. Again it follows that $\rho^{*}(y)$ attains its infimum, on $P$.

3. In this note we shall demonstrate an alternative method of resolving the dilemma. We begin by proving an inequality, which is of some intrinsic interest when applied to the characteristic vector $x$.

An inequality: Let $\mathrm{A}$ be an n-th order non-negative irreducible matrix, $\kappa a$ least diagonal element of $\mathrm{A}$, and $\lambda$ a least non-vanishing non-diagonal element. Let $y$ be a positive column vector, and suppose that $y_{1} \geqslant y_{2} \geqslant \ldots \geqslant y_{n}$. If $\rho^{*}(y) \leqslant \mathrm{M}$, then

$$
\frac{y_{n}}{y_{1}} \geqslant\left(\frac{\lambda}{M-\kappa}\right)^{n-1}
$$

Proof: Let $1 \leqslant k<n$. Then for all $i>k$,

$$
M y_{i} \geqslant \sum_{j=1}^{n} a_{i j} y_{j} \geqslant \sum_{j=1}^{k} a_{i j} y_{j}+a_{i i} y_{i}
$$

whence

$$
(M-\kappa) y_{k+1} \geqslant(M-\kappa) y_{i} \geqslant\left(\sum_{j=1}^{k} a_{i j}\right) y_{k}
$$

Since $A$ is irreducible, here is at least one $i>k$ for which $\sum_{j=1}^{k} a_{i j} \geqslant \lambda>0$. Hence it follows from (4) that

$$
\frac{y_{k+1}}{y_{k}} \geqslant \frac{\lambda}{M-\kappa}
$$

The inequality (3) is obtained from (5) by setting $k=1, \ldots, n-1$ and multiplying.

Corollary: If, in addition, $\Sigma_{i=1}^{n} y_{i}=1$, then for all $i$

$$
y_{i} \geqslant \frac{1}{n}\left(\frac{\lambda}{M-\kappa}\right)^{n-1} \text {. }
$$

For

$$
1=\sum_{i=1}^{n} y_{i} \leqslant n y_{1} \leqslant n\left(\frac{M-\kappa}{\lambda}\right)^{n-1} y_{n} .
$$

4. We now turn to the proof of the fundamental theorem. We shall consider $\rho^{*}(y)$ only. Set

and

$$
\begin{aligned}
& R=\max _{i} \sum_{j=1}^{n} a_{i j}, \\
& \delta=\frac{1}{n}\left(\frac{\lambda}{R-\kappa}\right)^{n-1} .
\end{aligned}
$$

Clearly $R \geqslant k+\lambda$, whence

$$
\delta \leqslant \frac{1}{n} \text {. }
$$


Let $\mathrm{P}_{2}$ be that part of the plane section of the non-negative cone defined by $\Sigma_{i=1}^{n} y_{i}=1$, and $y_{i} \geqslant \delta$, for all $i$. Evidently $P_{2}$ is bounded and closed, $\rho^{*}(y)$ is continuous everywhere on $P_{2}$, and hence $\rho^{*}(y)$ attains its infimum over $P_{2}$, say at the vector $x$ on $P_{2}$. Set $\rho=\rho^{*}(x)=\inf \rho^{*}(y)$ over $P_{2}$.

We note that $\rho$ is also the infimum of $\rho^{*}(y)$ over all positive $y$. It is sufficient to consider the infimum on the plane section $P_{1}$, and to show that there exists a $z$ on $P_{2}$ for which $\rho^{*}(z)<\rho^{*}(y)$ for all $y$ on $P_{1}$ which do not lie on $P_{2}$. By (7), the vector $z=\frac{1}{n}(1,1, \ldots, 1)$ belongs to $P_{2}$, while by $(6)$,

$$
\rho^{*}(y)>R=\rho^{*}(z) \geqslant \rho,
$$

if $y$ belongs to $P_{1}$ but not to $P_{2}$.

5. In this section we shall show that $\rho$ is a characteristic root of $A$, and that the positive vector $x$ is a characteristic vector belonging to $\rho$, viz. that $A x=\rho x$. We shall prove the equivalent proposition : If $z>0$ and $\rho_{*}(z)<\rho^{*}(z)$, then $\rho<\rho^{*}(z)$. Our proof is entirely due to Householder (2). Suppose that

$$
\begin{aligned}
\frac{(A z)_{i}}{z_{i}} & =\rho^{*}(z), \quad i=1, \ldots, k<n \\
\frac{(A z)_{i}}{z_{i}}<\rho^{*}(z), & i=k+1, \ldots, n .
\end{aligned}
$$

Since $A$ is irreducible there exists a positive element $a_{p q}$ with $1 \leqslant p \leqslant k$ and $k+1 \leqslant q \leqslant n$. Define the vector $z^{\prime}$ by setting $z_{i}^{\prime}=z_{i}$ if $i \neq q$ and $0<z_{q}^{\prime}<z_{q}$, where $z_{q}^{\prime}$ is chosen sufficiently close to $z_{q}$ to ensure that

$$
\frac{\left(A z^{\prime}\right)_{q}}{z_{q}^{\prime}}<\rho^{*}(z)
$$

But for all $i \neq q$

$$
\frac{\left(A z^{\prime}\right)_{i}}{z_{i}^{\prime}} \leqslant \frac{(A z)_{i}}{z_{i}}
$$

It follows from (9), (10), (11) and (12) that

$$
\rho^{*}\left(z^{\prime}\right) \leqslant \rho^{*}(z) \text {. }
$$

Since

$$
\frac{\left(A z^{\prime}\right)_{p}}{z_{p}^{\prime}}<\frac{(A z)_{p}}{z_{p}}
$$

the equality

$$
\frac{\left(A z^{\prime}\right)_{i}}{z_{i}^{\prime}}=\rho^{*}(z)
$$

can hold for at most $k-1$ indices $i$. Thus, by repetition of this process we may construct a vector $z^{\prime \prime}$ satisfying $\rho^{*}\left(z^{\prime \prime}\right)<\rho^{*}(x)$. We deduce that $\rho<\rho^{*}(z)$.

6. If $\rho$ is not a simple characteristic root of $A$, suppose first that $\rho$ has the linearly independent characteristic vectors $x>0$ and $z$ belonging to it. By choosing the real numbers $\alpha$ and $\beta$ suitably, we may obtain a positive characteristic vector $\alpha x+\beta z$ on $P_{i}$ belonging to $\rho$, which has some element less than $\delta$. But this is impossible, by (6) and (8). Next, suppose that $\rho$ is not simple, but has only one linearly independent characteristic vector belonging to it. 
Then there exists a column vector $y$ satisfying

$$
A y=\rho y-x,
$$

and replacing $y$ by $y+\gamma x$, it follows there exists a positive $y$ satisfying (13). For this $y, \rho^{*}(y)<\rho$, which is impossible. We have proved that $\rho$ is a simple characteristic root.

7. For the sake of completeness, we add a standard proof that $\rho$ is a maximal characteristic root. Let $\sigma$ be any characteristic root of $A$ and $u$ a row vector satisfying $u A=\sigma u$. Then, for all $j$,

$$
|\sigma|\left|u_{j}\right|=\left|\sum_{i=1}^{n} u_{i} a_{i j}\right| \leqslant \sum_{i=1}^{n}\left|u_{i}\right| \alpha_{i j}
$$

whence

and so

$$
|\sigma| \Sigma_{j=1}^{n}\left|u_{j}\right| x_{j} \leqslant \sum_{i, j=1}^{n}\left|u_{i}\right| a_{i j} x_{j}=\rho \sum_{i=1}^{n}\left|u_{i}\right| x_{i} \text {, }
$$

since

$$
|\sigma| \leqslant \rho
$$

$$
\sum_{i=1}^{n}\left|u_{i}\right| x_{i}>0 .
$$

8. The inequality (3) leads to a positive lower bound for the ratios of the elements of the characteristic vector $x$. In view of $\rho^{*}(x)=\rho$ and (8) it follows that

$$
\frac{\min _{i} x_{i}}{\max _{i} x_{i}} \geqslant\left(\frac{\lambda}{\rho-\kappa}\right)^{n-1} \geqslant\left(\frac{\lambda}{R-\kappa}\right)^{n-1}
$$

For the case $A>0$, lower bounds for the ratios $x_{j} / x_{i}$ have already been found by Ostrowski (3) and A. Brauer (1). These bounds, however, reduce to 0 when $A$ has zero elements.

\section{REFERENCES}

(1) A. Brater, The theorems of Ledermann and Ostrowski on positive matrices, Duke Math. J., 24 (1957), 265-274.

(2) A. S. Housenolder, On the convergence of matrix iterations, Oak Ridge National Laboratory, Physics No. 1883 (1955).

(3) A. OsmrowskI, Bounds for the greatest latent root of a positive matrix, J. London Math. Soc., 27 (1952), 253.256.

(4) H. WIELANDT, Unzerlegbare, nicht negative Matrizen, Math. Zeitschrift, $52(1950), 642-8$.

\section{The Queen's UnIversity}

BeLfast 